\title{
Randomized Comparison of Anatomic and Electrogram Mapping Approaches to Ablation of Typical Atrial Flutter
}

\author{
HIROSHI TADA, M.D., HAKAN ORAL, M.D., MEHMET OZAYDIN, M.D., \\ AMAN CHUGH, M.D., CHRISTOPH SCHARF, M.D., SOHAIL HASSAN, M.D., \\ RADMIRA GREENSTEIN, M.D., FRANK PELOSI, JR., M.D., BRADLEY P. KNIGHT, M.D., \\ S. ADAM STRICKBERGER, M.D., and FRED MORADY, M.D.
}

From the Division of Cardiology, Department of Internal Medicine, University of Michigan, Ann Arbor, Michigan

\begin{abstract}
Isthmus Ablation. Introduction: The cavotricuspid isthmus can be ablated using an anatomic approach or an electrogram mapping approach in which sites at which there is a gap in the line of block are targeted. The aim of this study was to compare the anatomic and electrogram mapping approaches for creating a line of block in the cavotricuspid isthmus after an initial, unsuccessful anatomically directed ablation line.

Methods and Results: The subjects of this study were 63 patients with isthmus-dependent atrial flutter in whom a single series of contiguous applications of radiofrequency energy guided by fluoroscopy in the cavotricuspid isthmus did not result in complete block. The patients were randomly assigned to additional ablation on an anatomic basis $(n=31)$ or guided by single potentials or narrowly split double potentials during coronary sinus pacing $(n=32)$. After every 15 applications of radiofrequency energy, the alternate approach was used until complete block was achieved. Before cross-over, complete block was achieved in 6 patients $(19 \%)$ with the anatomic approach compared with 19 patients $(59 \%)$ with the electrogram mapping approach $(P<0.005)$. The electrogram mapping approach also was more effective than the anatomic approach in achieving complete isthmus block after the first cross-over $(72 \%$ vs $23 \%, P<0.005)$ and after the second cross-over $(80 \%$ vs $42 \%, P<0.05)$.

Conclusion: When there is incomplete block after an initial series of applications of radiofrequency energy in the cavotricuspid isthmus, complete block is achieved more efficiently with an electrogram mapping approach than with an anatomic approach. (J Cardiovasc Electrophysiol, Vol. 13, pp. 662-666, July 2002)
\end{abstract}

atrial flutter, cavotricuspid isthmus, radiofrequency catheter ablation

\section{Introduction}

The goal of radiofrequency ablation of isthmus-dependent atrial flutter is to create a line of complete block in the cavotricuspid isthmus. This can be achieved with an anatomic approach to delivery of radiofrequency energy in the cavotricuspid isthmus, guided by fluoroscopy..$^{1-8}$ An alternative approach is to target only the sites in the cavotricuspid isthmus where there is incomplete block, guided by characteristics of the local atrial electrograms. ${ }^{7,9}$ These two approaches have not been compared. Therefore, the aim of this prospective, randomized study was to compare the anatomic and electrogram approaches for ablation of the cavotricuspid isthmus in patients in whom an initial, anatomically directed ablation line was unsuccessful in creating isthmus block.

\section{Methods}

\section{Subject Characteristics}

The subjects of this study were 63 consecutive patients who had isthmus-dependent atrial flutter and in whom a

Supported in part by a grant from the Gunma Prefecture Government and the Ellen and Robert Thompson Atrial Fibrillation Research Fund Isthmus Ablation.

Address for correspondence: Fred Morady, M.D., Division of Cardiology, Department of Internal Medicine, University of Michigan, 1500 East Medical Center Drive, B1F245, Ann Arbor, MI 48109-0022. Fax: 734936-7026; E-mail: fmorady@umich.edu

Manuscript received 4 April 2002; Accepted for publication 7 May 2002. single series of contiguous applications of radiofrequency energy in the cavotricuspid isthmus did not result in complete block. Nine patients who underwent catheter ablation during the same period of time as the subjects of this study and in whom a line of complete block was achieved after a single series of applications in the cavotricuspid isthmus were excluded. There were 47 men and 16 women (mean age $55 \pm 13$ years). Mean duration of symptomatic atrial flutter was $5.0 \pm 6.4$ years. The atrial flutter was chronic in 30 patients and paroxysmal in 33 patients. Structural heart disease was present in 13 patients $(21 \%)$ : coronary heart disease in 5 , nonischemic cardiomyopathy in 4 , hypertensive heart disease in 2, and valvular heart disease in 2 . Echocardiography demonstrated mean left ventricular ejection fraction of $0.53 \pm 0.06$ and mean left atrial diameter of $42 \pm 4.7 \mathrm{~mm}$.

\section{Electrophysiologic Procedure}

The ablation procedures were performed at least five half-lives after discontinuation of antiarrhythmic drug therapy, except in the case of amiodarone, which was discontinued 1 to 2 weeks before the procedure. The study protocol was approved by the Institutional Review Board. After informed consent was obtained, three catheters were inserted into a femoral vein. A 7-French 20-pole catheter (spacing between electrodes of each bipole: $2 \mathrm{~mm}$; spacing between pairs of electrodes: 20-2-2-2-2-2-2-25-25-25 mm; Daig Corporation, Minnetonka, MN, USA) was positioned along the tricuspid annulus, straddling the cavotricuspid 
TABLE 1

Characteristics of Subjects Randomly Assigned to the Anatomic and Electrogram Mapping Approaches

\begin{tabular}{lccc}
\hline & $\begin{array}{c}\text { Anatomic } \\
(\boldsymbol{n}=\mathbf{3 1})\end{array}$ & $\begin{array}{c}\text { Electrogram } \\
\text { Mapping } \\
(\boldsymbol{n}=\mathbf{3 2})\end{array}$ & $\begin{array}{c}\mathbf{P} \\
\text { Value }\end{array}$ \\
\hline Age (years) & $56 \pm 11$ & $55 \pm 16$ & 0.8 \\
Male/female & $22 / 9$ & $24 / 7$ & 0.6 \\
Structural heart disease & $7(22 \%)$ & $7(22 \%)$ & 0.9 \\
Atrial flutter during procedure & $13(42 \%)$ & $9(28 \%)$ & 0.3 \\
Left atrial diameter (mm) & $42 \pm 4.9$ & $42 \pm 4.6$ & 0.7 \\
Therapy with amiodarone & $19(61 \%)$ & $21(66 \%)$ & 0.7 \\
\hline
\end{tabular}

isthmus, with the distal bipole of the catheter near the ostium of the coronary sinus. A 7-French quadripolar catheter (EP Technologies, San Jose, CA, USA) was positioned within the proximal coronary sinus for pacing. A 7-French quadripolar catheter with a deflectable tip, 4-mm distal electrode, 2-5-2 mm interelectrode spacing, and a thermistor embedded in the distal electrode (EP Technologies) was used for mapping the isthmus and delivering radiofrequency energy.

Bipolar electrograms were recorded with the distal electrode serving as the cathode, filtered at settings of 30 to 500 $\mathrm{Hz}$, and recorded digitally (EPMed Systems, Mount Arlington, NJ, USA).

\section{Radiofrequency Ablation}

Radiofrequency ablation was performed during atrial flutter in 22 patients and during coronary sinus pacing at a cycle length of $600 \mathrm{msec}$ in 41 patients who were in sinus rhythm. Radiofrequency energy was delivered at single sites in the cavotricuspid isthmus using a conventional ablation catheter. The power was automatically adjusted to maintain a target temperature of $60^{\circ} \mathrm{C}$ at the electrode-tissue interface. Each application of energy was 50 to 60 seconds in duration.

\section{Study Protocol}

The first ablation line was created in the isthmus under fluoroscopic guidance, at the 5- to 6-o'clock position relative to the tricuspid annulus on a $45^{\circ}$ to $60^{\circ}$ left anterior oblique view. In accordance with the selection criteria, none of the patients had complete isthmus block after the first ablation line. The patients were randomly assigned to further ablation based on an anatomic approach (31 patients) or on an electrogram mapping approach (32 patients). There were no significant differences in the demographic or clinical characteristics of the patients in the two groups (Table 1).

The anatomic approach consisted of the delivery of discrete, contiguous applications of radiofrequency energy, starting at the tricuspid annulus and ending at the inferior vena cava, under fluoroscopic guidance and along the original ablation line.

In the electrogram mapping approach group, discrete applications of radiofrequency energy were delivered at sites along the original ablation line where the atrial potential during coronary sinus pacing was single or narrowly split, with a double-potential interval $\leq 90 \mathrm{msec}$ (Fig. 1). ${ }^{10}$

After randomization, up to 15 applications of radiofrequency energy were delivered using either the anatomic or the electrogram mapping approach. If complete block was not achieved, up to 15 additional applications of radiofrequency energy were delivered along the same ablation line using the alternate approach (first cross-over). If there still was not complete block in the cavotricuspid isthmus, up to 15 more applications of radiofrequency energy were delivered along the same ablation line using the original approach (second cross-over).

With both approaches, the endpoint of ablation was complete block in the cavotricuspid isthmus. The criteria for complete block were assessed during coronary sinus pacing and consisted of an appropriate atrial activation sequence recorded with the 20-pole catheter, negative polarity of the bipolar electrograms recorded just lateral to the ablation line, ${ }^{11}$ and double potentials with a double-potential interval $>110 \mathrm{msec}$ or an isoelectric double-potential interval $>90$ msec along the entire ablation line (Fig. 2). ${ }^{12}$ These criteria also had to be present during infusion of 1 to $4 \mu \mathrm{g} / \mathrm{min}$ of isoproterenol, titrated to maintain a sinus rate of $120 / \mathrm{min}$.

If complete isthmus block was not achieved after the second cross-over, additional applications of energy were delivered along a new line in the cavotricuspid isthmus, with the technique being at the discretion of the operator.

\section{Follow-Up}

The procedures were performed on an outpatient basis. Antiarrhythmic drug therapy was reinstituted after the ablation procedure in 24 patients who were known to also have atrial fibrillation. The patients were seen in follow-up every 3 to 4 months. An event monitor was provided to patients who described symptoms suggestive of recurrent atrial flutter.

\section{Statistical Analysis}

Continuous variables are expressed as mean $\pm 1 \mathrm{SD}$. The clinical characteristics of the patients in the anatomic and electrogram mapping groups were compared with Student's $t$-test or by Chi-square analysis. The success rates in achieving complete isthmus block with the two approaches were compared by Chi-square analysis or with the Fisher's exact test. The procedure time, duration of fluoroscopy, and duration of radiofrequency energy needed to achieve isthmus block with the two approaches were compared using Student's $t$-test. $\mathrm{P}<0.05$ were considered statistically significant.
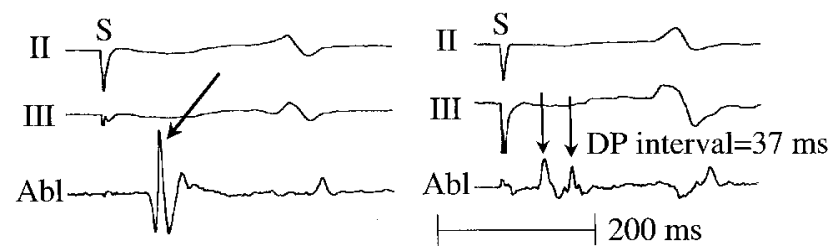

Figure 1. Examples of the criteria used to select target sites for ablation in the electrogram mapping approach. Displayed are leads II and III and electrograms recorded by the ablation catheter (Abl). Applications of energy were delivered at sites along the ablation line where the atrial electrograms (arrows) during coronary sinus pacing were either single potentials (left) or double potentials with an interval $<90 \mathrm{~ms}$ between the two components (right). DP $=$ double potential; $S=$ stimulus. 


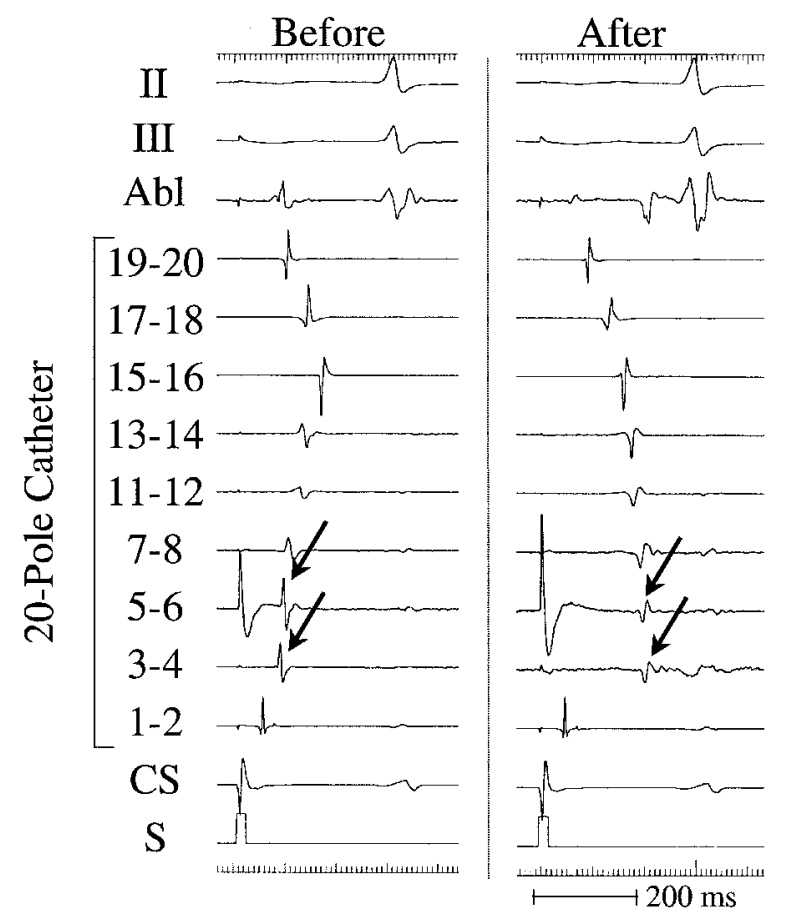

Figure 2. Examples of the criteria for complete isthmus block. Shown are leads II and III, a recording obtained with the ablation catheter ( $\mathrm{Abl})$ positioned on the ablation line in the cavotricuspid isthmus, recordings from the 20-pole catheter, a coronary sinus electrogram (CS), and a stimulus channel $(S)$. Electrodes 3-4 and 5-6 were positioned just lateral to the ablation line. The recordings were obtained during coronary sinus pacing. Before complete isthmus block, the atrial activation pattern recorded with the 20-pole catheter was consistent with conduction across the cavotricuspid isthmus, the initial electrogram polarity was positive at electrodes 3-4 and 5-6 of the 20-pole catheter (arrows), and the atrial potential recorded on the ablation line was single. After complete isthmus block, the activation sequence was consistent with conduction only in the counterclockwise direction around the tricuspid annulus, the initial electrogram polarity became negative at electrodes 3-4 and 5-6, and there was a double potential with an interval of $125 \mathrm{~ms}$ between the two components, at this site along the ablation line. Mapping demonstrated a widely split double potential separated by an interval $>110 \mathrm{~ms}$ throughout the ablation line (not shown).

\section{Results}

\section{Results After Randomization}

After randomization, complete isthmus block was achieved with $\leq 15$ applications of radiofrequency energy in 6 patients (19\%) using the anatomic approach and in 19 patients $(59 \%)$ using the electrogram mapping approach $(\mathrm{P}<0.005)$. The procedure time was significantly longer in patients in the electrogram mapping group than in the patients in the anatomic group $(\mathrm{P}<0.05$, Table 2$)$. However, the total duration of radiofrequency energy was significantly shorter in patients who underwent ablation with the electrogram mapping approach than with the anatomic approach $(\mathrm{P}<0.05$, Table 2).

\section{Results After First Cross-Over}

After the first cross-over, complete isthmus block was achieved using the electrogram mapping approach in 18 (72\%) of the 25 patients in whom the anatomic approach had been ineffective. Complete isthmus block was achieved
TABLE 2

Procedural Endpoints After Initial Randomization to the Anatomic and Electrogram Mapping Approaches

\begin{tabular}{lccc}
\hline & $\begin{array}{c}\text { Anatomic } \\
(\boldsymbol{n}=\mathbf{3 1})\end{array}$ & $\begin{array}{c}\text { Electrogram } \\
\text { Mapping } \\
(\boldsymbol{n}=\mathbf{3 1})\end{array}$ & $\begin{array}{c}\text { P } \\
\text { Value }\end{array}$ \\
\hline Complete block & $6(19 \%)$ & $19(59 \%)$ & $<0.005$ \\
Procedure duration (min) & $13.2 \pm 6.3$ & $27.3 \pm 13.0$ & $<0.05$ \\
$\begin{array}{l}\text { Fluoroscopy time (min) } \\
\begin{array}{l}\text { Total duration of } \\
\text { radiofrequency energy (min) }\end{array}\end{array}$ & $5.3 \pm 1.9$ & $10.2 \pm 6.7$ & 0.1 \\
\hline \multicolumn{2}{l}{ Values expressed as mean \pm 1 SD. } & & \\
\end{tabular}

using the anatomic approach in $3(23 \%)$ of the 13 patients in whom the electrogram mapping approach had been ineffective $(\mathrm{P}<0.005)$.

Among the patients in whom complete block was achieved, the procedure time, fluoroscopy time, and total duration of radiofrequency energy did not differ significantly between the anatomic and electrogram mapping approaches (Table 3 ).

\section{Results After Second Cross-Over}

After the second cross-over, complete block was achieved in $3(42 \%)$ of 7 patients using the anatomic approach compared with $8(80 \%)$ of 10 patients using the electrogram mapping approach $(\mathrm{P}<0.05)$. Among the patients in whom complete block was achieved, the procedure time was significantly shorter in patients in the electrogram mapping group than in the patients in the anatomic group $(\mathrm{P}<0.05$, Table 4$)$. However, there were no significant differences in fluoroscopy time and total duration of radiofrequency energy between the two approaches (Table 4).

\section{Patients with Incomplete Block After Second Cross-Over}

Including the first and second cross-overs, complete block was achieved in a total of 57 (90\%) of the 63 randomized patients. Complete isthmus block was not achieved after the second cross-over in four patients who had been randomly assigned to the anatomic approach and in two patients who had been randomly assigned to the electrogram mapping approach. Complete block was achieved in these patients with an additional ablation line at another site in the cavotricuspid isthmus.

\section{Follow-Up}

During a mean of $16.5 \pm 7.7$ months of follow-up, 1 (2\%) of 31 patients randomly assigned to the anatomic

TABLE 3

Procedural Endpoints After First Cross-Over to the Anatomic and Electrogram Mapping Approaches

\begin{tabular}{lccc}
\hline & $\begin{array}{c}\text { Anatomic } \\
(\boldsymbol{n}=\mathbf{1 3})\end{array}$ & $\begin{array}{c}\text { Electrogram } \\
\text { Mapping } \\
(\boldsymbol{n}=\mathbf{2 5})\end{array}$ & $\begin{array}{c}\mathbf{P} \\
\text { Value }\end{array}$ \\
\hline Complete block & $3(23 \%)$ & $18(72 \%)$ & $<0.005$ \\
Procedure duration (min) & $34.3 \pm 20.0$ & $20.4 \pm 11.7$ & 0.1 \\
$\begin{array}{l}\text { Fluoroscopy time (min) } \\
\begin{array}{l}\text { Total duration of } \\
\text { radiofrequency energy (min) }\end{array}\end{array}$ & $15.4 \pm 7.1$ & $8.9 \pm 6.1$ & 0.1 \\
\hline Values & $11.9 \pm 4.6$ & $6.9 \pm 5.3$ & 0.1 \\
\hline
\end{tabular}

Values expressed as mean $\pm 1 \mathrm{SD}$. 
TABLE 4

Procedural Endpoints After Second Cross-Over to the Anatomic and Electrogram Mapping Approaches

\begin{tabular}{lccc}
\hline & $\begin{array}{c}\text { Anatomic } \\
(\boldsymbol{n}=\mathbf{7})\end{array}$ & $\begin{array}{c}\text { Electrogram } \\
\text { Mapping } \\
(\boldsymbol{n}=\mathbf{1 0})\end{array}$ & $\begin{array}{c}\mathbf{P} \\
\text { Value }\end{array}$ \\
\hline Complete block & $3(43 \%)$ & $8(80 \%)$ & $<0.05$ \\
Procedure duration (min) & $34.0 \pm 6.1$ & $15.8 \pm 12.2$ & $<0.05$ \\
$\begin{array}{l}\text { Fluoroscopy time (min) } \\
\begin{array}{c}\text { Total duration of } \\
\text { radiofrequency energy (min) }\end{array}\end{array}$ & $11.1 \pm 4.2$ & $6.7 \pm 6.0$ & 0.3 \\
\hline Values expressed as mean $\pm 1 \mathrm{SD}$. & $8.3 \pm 5.1$ & 0.3 \\
\hline
\end{tabular}

group had a recurrence of atrial flutter compared with 2 $(6 \%)$ of the 32 patients randomly assigned to the electrogram mapping approach $(\mathrm{P}=0.7)$.

\section{Discussion}

\section{Main Findings}

The results of this study demonstrate that when there is persistent conduction across the cavotricuspid isthmus after an initial series of applications of radiofrequency energy, complete isthmus may be achieved more efficiently with an electrogram approach than with an anatomic approach. Identification of sites along the original ablation line at which the atrial electrogram during atrial flutter or coronary sinus pacing was single or narrowly split resulted in complete isthmus block with $\leq 15$ additional conventional applications of radiofrequency energy three times more often than when a fluoroscopically guided anatomic approach was used. When complete isthmus block was not achieved with the approach to which the patient had been randomly assigned, the greater efficiency of the electrogram mapping approach also was apparent after cross-over.

\section{Anatomy of the Cavotricuspid Isthmus}

The anatomic characteristics of the cavotricuspid isthmus may at least partially explain why an electrogram mapping approach was found to be more efficient than an anatomic approach in creating complete isthmus block in this study. First, there are variable amounts of fibrous tissue and muscular defects in the cavotricuspid isthmus, implying that a continuous ablation line across the cavotricuspid isthmus sometimes may not be necessary. ${ }^{13,14}$ Consistent with these anatomic findings, a prior study demonstrated that patients with typical atrial flutter may have partial block in the cavotricuspid isthmus even before any attempts at ablation, and that unnecessary applications of radiofrequency energy were avoided by using an electrogram mapping approach. ${ }^{15}$ Therefore, a purely anatomic approach that ignores the characteristics of the local electrograms may result in the delivery of radiofrequency energy to sites along the isthmus that are void of muscle and that do not need to be ablated.

A second anatomic feature of the cavotricuspid isthmus is the frequent presence of recesses or a pouch along its floor. ${ }^{13,16,17}$ With the anatomic approach, in which the ablation catheter is withdrawn from the tricuspid valve to the inferior vena cava, it may be less likely that the distal electrode of the catheter will enter these recesses or pouches than with the electrogram mapping approach, in which the ablation line typically is mapped by both advancing and withdrawing the ablation catheter along the cavotricuspid isthmus. This provides another advantage of the electrogram mapping approach over the anatomic approach in achieving complete isthmus block with the fewest applications of energy.

\section{Anatomic Approach}

In this study, the anatomic approach consisted of discrete, contiguous applications of radiofrequency energy along the cavotricuspid isthmus, as described in prior studies..$^{1,2,8}$ Another technique used has been pullback of the ablation catheter along the cavotricuspid isthmus during continuous application of energy. ${ }^{3-7}$ Although the two techniques have not been directly compared, similar results have been reported with both ${ }^{1-8}$; therefore, it is unlikely that either technique has a major advantage over the other. In this study, discrete applications of energy were used to facilitate comparison of the anatomic and the electrogram mapping approach using a uniform endpoint of 15 applications with both techniques.

In prior studies, the efficacy of an anatomic approach to ablation using a conventional ablation catheter has been quite variable, ranging from success rates as low as $54 \%$ to $67 \%{ }^{6,18}$ to as high as $97 \%$ to $100 \% .^{3,5}$ Several factors, both patient and operator dependent, may explain this wide range of success rates. Nevertheless, it is clear that a high success rate is potentially achievable with the anatomic approach. Therefore, the results of the present study should not be interpreted as demonstrating that the electrogram mapping approach results in complete isthmus block in a higher percentage of patients than the anatomic approach. Instead, the results demonstrate that the electrogram mapping approach is more efficient than the anatomic approach, more often resulting in complete isthmus block with $\leq 15$ applications of radiofrequency energy.

\section{Electrogram Mapping Approach}

Complete isthmus block has been demonstrated to be associated with a parallel corridor of double potentials along the entire ablation line..$^{10,12}$ When there are gaps in the ablation line, the local atrial electrogram is single, fractionated, or narrowly split. ${ }^{10,12}$ This provides the basis for the electrogram mapping approach, in which radiofrequency energy is delivered only at sites at which widely split double potentials are not present. $7,9,10,12$

\section{Prior Studies}

A prior study compared an anatomic approach for creating block in the cavotricuspid isthmus with an electrophysiologically guided approach. ${ }^{3}$ However, in that study, ablation sites in the electrophysiologically guided group were selected based on concealed entrainment and the postpacing interval, not using the criterion of single or narrowly split atrial potentials that was used in the present study. Nevertheless, some of the results of the two studies are similar. For example, in both studies, the procedure time and duration of fluoroscopy were approximately 50\% shorter when the anatomic approach was used than when the electrophysiologically guided approach was used. This reflects the extra time needed to identify specific target sites in the cavotricuspid isthmus when using an electrophysiologically guided approach. 


\section{Study Limitations}

A limitation of this study is that there were two crossover phases if the primary approach that was randomly chosen was unsuccessful in creating complete isthmus block with $\leq 15$ applications of energy. Therefore, both the anatomic and electrogram mapping approaches were used in some patients, and it is possible that both of the approaches contributed to an eventually successful outcome, regardless of the particular approach that was being used at the time that isthmus block was achieved. For this reason, comparisons of the two approaches after cross-over may not be as valid as the comparison before cross-over.

Another limitation of this study is that the results apply only to radiofrequency ablation using a conventional catheter that has a 4-mm distal electrode. Use of an ablation catheter with an 8- to $10-\mathrm{mm}$ distal electrode ${ }^{18,19}$ or an irrigated tip, ${ }^{20,21}$ because of a larger lesion size, may have mitigated the differences between the anatomic and electrogram mapping approaches that were found in this study.

A third limitation of this study is that the anatomic approach was guided only by fluoroscopy. It is possible that the anatomic approach would have been more effective under the guidance of intracardiac echocardiography $y^{22}$ or electroanatomic mapping. . $^{23,24}$

\section{Conclusion}

In the majority of patients with isthmus-dependent atrial flutter, ablation along the entire cavotricuspid isthmus is required. ${ }^{15}$ In these patients, an anatomic approach guided by fluoroscopy or by a more sophisticated catheter locator system is appropriate. However, using conventional radiofrequency catheter ablation, the first series of energy applications in the cavotricuspid isthmus resulted in complete block in only $13 \%$ of patients in this study and in only $25 \%$ of patients in a prior study. ${ }^{17}$ Therefore, after the first ablation line, the operator often will have the option of either repeating the anatomic approach until complete block is achieved or using an electrogram mapping approach to specifically target gaps in the ablation line. Although a high success rate eventually can be achieved with either approach, the results of this study demonstrate that complete isthmus block can be achieved with fewer applications of energy with the electrogram mapping approach.

\section{References}

1. Kirkorian G, Moncada E, Chevalier P, Canu G, Claudel JP, Bellon C, Lyon L, Touboul P: Radiofrequency ablation of atrial flutter. Efficacy of an anatomically guided approach. Circulation 1994;90:2804-2814.

2. Fischer B, Haissaguerre M, Garrigues S, Poquet F, Gencel L, Clementy J, Marcus FI: Radiofrequency catheter ablation of common atrial flutter in 80 patients. J Am Coll Cardiol 1995;25:1365-1372.

3. Chen SA, Chiang CE, Wu TJ, Tai CT, Lee SH, Cheng CC, Chiou CW, Ueng KC, Wen ZC, Chang MS: Radiofrequency catheter ablation of common atrial flutter: Comparison of electrophysiologically guided focal ablation technique and linear ablation technique. J Am Coll Cardiol 1996;27:860-868.

4. Poty H, Saoudi N, Nair M, Anselme F, Letac B: Radiofrequency catheter ablation of atrial flutter. Further insights into the various types of isthmus block: Application to ablation during sinus rhythm. Circulation 1996;94:3204-3213.

5. Schwartzman D, Callans DJ, Gottlieb CD, Dillon SM, Movsowitz C, Marchlinski FE: Conduction block in the inferior vena caval-tricuspid valve isthmus: Association with outcome of radiofrequency ablation of type I atrial flutter. J Am Coll Cardiol 1996;28:1519-1531.

6. Schumacher B, Pfeiffer D, Tebbenjohanns J, Lewalter T, Jung W, Luderitz B: Acute and long-term effects of consecutive radiofrequency applications on conduction properties of the subeustachian isthmus in type I atrial flutter. J Cardiovasc Electrophysiol 1998;9:152-163.

7. Chen J, de Chillou C, Basiouny T, Sadoul N, Filho JD, Magnin-Poull I, Messier M, Aliot E: Cavotricuspid isthmus mapping to assess bidirectional block during common atrial flutter radiofrequency ablation. Circulation 1999;100:2507-2513.

8. Paydak H, Kall JG, Burke MC, Rubenstein D, Kopp DE, Verdino RJ, Wilber DJ: Atrial fibrillation after radiofrequency ablation of type I atrial flutter: Time to onset, determinants, and clinical course. Circulation 1998;98:315-322.

9. Shah DC, Haissaguerre M, Jais P, Fischer B, Takahashi A, Hocini M, Clementy J: Simplified electrophysiologically directed catheter ablation of recurrent common atrial flutter. Circulation 1997:96:25052508.

10. Shah DC, Takahashi A, Jais P, Hocini M, Clementy J, Haissaguerre M: Local electrogram-based criteria of cavotricuspid isthmus block. J Cardiovasc Electrophysiol 1999;10:662-669.

11. Tada H, Oral H, Sticherling C, Chough SP, Baker RL, Wasmer K, Kim MH, Pelosi F Jr, Michaud GF, Knight BP, Strickberger SA, Morady F: Electrogram polarity and cavotricuspid isthmus block during ablation of typical atrial flutter. J Cardiovasc Electrophysiol 2001;12:393-399.

12. Tada H, Oral H, Sticherling C, Chough SP, Baker RL, Wasmer K, Pelosi F Jr, Knight BP, Strickberger SA, Morady F: Double potentials along the ablation line as a guide to radiofrequency ablation of typical atrial flutter. J Am Coll Cardiol 2001;38:750-755.

13. Cabrera JA, Sanchez-Quintana D, Ho SY, Medina A, Anderson RH: The architecture of the atrial musculature between the orifice of the inferior caval vein and the tricuspid valve: The anatomy of the isthmus. J Cardiovasc Electrophysiol 1998;9:1186-1195.

14. Waki K, Saito T, Becker AE: Right atrial flutter isthmus revisited: Normal anatomy favors nonuniform anisotropic conduction. J Cardiovasc Electrophysiol 2000;11:90-94.

15. Takahashi A, Shah DC, Jais P, Hocini M, Clementy J, Haissaguerre M: Partial cavotricuspid isthmus block before ablation in patients with typical atrial flutter. J Am Coll Cardiol 1999;33:1996-2002.

16. Cabrera JA, Sanchez-Quintana D, Ho SY, Medina A, Wanguemert F, Gross E, Grillo J, Hernandez E, Anderson RH: Angiographic anatomy of the inferior right atrial isthmus in patients with and without history of common atrial flutter. Circulation 1999;99:3017-3023.

17. Heidbuchel H, Willems R, van Rensburg H, Adams J, Ector H, Van de Werf F: Right atrial angiographic evaluation of the posterior isthmus: Relevance for ablation of typical atrial flutter. Circulation 2000;101: 2178-2184.

18. Tsai CF, Tai CT, Yu WC, Chen YJ, Hsieh MH, Chiang CE, Ding YA, Chang MS, Chen SA: Is 8-mm more effective than 4-mm tip electrode catheter for ablation of typical atrial flutter? Circulation 1999;100:768771.

19. Saxon LA, Kalman JM, Olgin JE, Scheinman MM, Lee RJ, Lesh MD: Results of radiofrequency catheter ablation for atrial flutter. Am J Cardiol 1996;77:1014-1016.

20. Jais P, Haissaguerre M, Shah DC, Takahashi A, Hocini M, Lavergne T, Lafitte S, Le Mouroux A, Fischer B, Clementy J: Successful irrigated-tip catheter ablation of atrial flutter resistant to conventional radiofrequency ablation. Circulation 1998;98:835-838.

21. Jais P, Shah DC, Haissaguerre M, Hocini M, Garrigue S, Le Metayer $\mathrm{P}$, Clementy J: Prospective randomized comparison of irrigated-tip versus conventional-tip catheters for ablation of common flutter. Circulation 2000;101:772-776.

22. Chu E, Kalman JM, Kwasman MA, Jue JC, Fitzgerald PJ, Epstein LM, Schiller NB, Yock PG, Lesh MD: Intracardiac echocardiography during radiofrequency catheter ablation of cardiac arrhythmias in humans. J Am Coll Cardiol 1994:24:1351-1357.

23. Shah DC, Jais P, Haissaguerre M, Chouairi S, Takahashi A, Hocini M, Garrigue S, Clementy J: Three-dimensional mapping of the common atrial flutter circuit in the right atrium. Circulation 1997;96:3904-3912.

24. Kottkamp H, Hugl B, Krauss B, Wetzel U, Fleck A, Schuler G, Hindricks G: Electromagnetic versus fluoroscopic mapping of the inferior isthmus for ablation of typical atrial flutter: A prospective randomized study. Circulation 2000;102:2082-2086 